\title{
Herpes zoster and its neurological complications
}

\author{
C.M. Chang, E. Woo, Y.L. Yu, C.Y. Huang and D. Chin \\ Department of Medicine, Queen Mary Hospital, University of Hong Kong, Hong Kong.
}

\begin{abstract}
Summary: Ninety-three Chinese patients with cutaneous herpes zoster were seen during a 4-year period. Thoracic zoster occurred most commonly, followed by ophthalmic, cervical and lumbosacral zoster. Neurological complications were present in eleven patients $(11.8 \%)$, the commonest being Ramsay-Hunt syndrome and segmental limb paresis. The clinical picture, pathogenesis, treatment and outcome of segmental limb paresis, myelitis and delayed contralateral hemiparesis following zoster ophthalmicus are discussed. Nine immunocompromised patients received intravenous adenine arabinoside (vidarabine) or acycloguanosine (acyclovir), and no cutaneous or visceral spread occurred in these patients.
\end{abstract}

\section{Introduction}

Herpes zoster infection is a common condition with predominantly cutaneous involvement along the dermatomes. Neurological complications beyond the posterior root ganglia and the first-order sensory neurones are infrequent. We undertook a retrospective analysis of the pattern of cutaneous involvement, the neurological complications and their outcome in 93 Chinese patients seen over a 4-year period.

\section{Materials and methods}

Patients under the care of the University Medical Unit, Queen Mary Hospital, Hong Kong between January 1981 and December 1984 with a diagnosis of herpes zoster were reviewed, with particular emphasis on the nature of any underlying disorder, the pattern of cutaneous involvement and the neurological complications. Those patients with definite motor complications were reassessed to determine their prognostic outcome. The use of intravenous adenine arabinoside (vidarabine) or acycloguanosine (acyclovir) for herpes zoster in 9 immunocompromised patients was also evaluated.

\section{Results}

Ninety-three patients were identified with a diagnosis of herpes zoster, and their age and sex distribution are shown in Figure 1. There were 34 males and 59

Correspondence: E. Woo, M.B., B.S.(H.K.), M.R.C.P. (U.K.)

Accepted: 10 September 1986 females. Their median age was 57 years with a range of 13 to 96 years; $69 \%$ of them were aged 50 or above. Eighty-five patients were hospitalized on account of the herpes zoster; the other eight $(8.6 \%)$ patients developed shingles while in hospital for another medical illness.

Twenty-one patients $(22.6 \%)$ had underlying diseases (Table I), mainly haematological malignancy, diabetes mellitus and systemic lupus erythematosus (SLE). Twelve of these 21 patients, including all 5 with SLE, were on steroid therapy at the time of the zoster infection.

The pattern of cutaneous involvement is shown in Table II. The thoracic dermatomes were most commonly affected, followed by the ophthalmic division of the trigeminal nerve, and the cervical and lumbosacral dermatomes.

Table I Underlying diseases in 21 patients with cutaneous herpes zoster

\begin{tabular}{lr}
\hline & Number \\
\hline Hodgkin's lymphoma & 3 \\
Non-Hodgkin's lymphoma & 1 \\
Leukaemia & 1 \\
Myeloproliferative disorder & 1 \\
Nasopharyngeal carcinoma & 1 \\
Bronchogenic carcinoma & 1 \\
Carcinoma of bladder & 1 \\
Diabetes mellitus & 5 \\
System lupus erythematosus (on steroids) & 5 \\
Chronic obstructive airway disease (on steroids) & 1 \\
Weber-Christian disease (on steroids) & 1
\end{tabular}




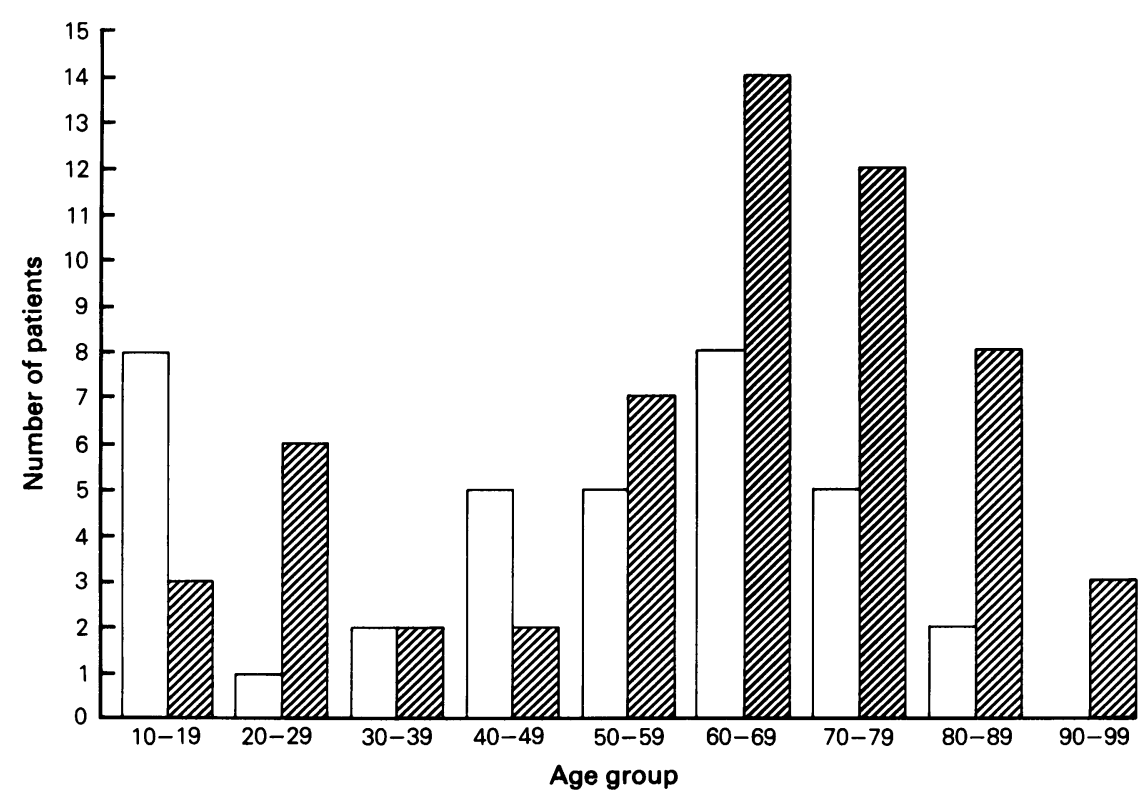

Figure 1 Age and sex distribution of 93 patients with herpes zoster; $\square$ male; $\mathbb{Z}$ female.

Neurological complications occurred in 11 patients $(11.8 \%)$ and the clinical data of these patients are presented in Table III. There were 3 patients with Ramsay-Hunt syndrome, 3 with segmental limb paresis, 2 with myelitis and 1 each with delayed contralateral hemiparesis, third nerve palsy and meningitis. Underlying diseases were present in 4 of these 11 patients.

The interval between the cutaneous zoster and the neurological complication ranged from simultaneous presentation to two and a half weeks, and was within one week in the majority of cases. In no patient did the neurological complication precede the cutaneous manifestation. The neurological complication evolved

Table II Cutaneous distribution of herpes zoster in 93 patients

\begin{tabular}{llc}
\hline & & $\begin{array}{c}\text { Number of } \\
\text { cases }\end{array}$ \\
\hline Trigeminal & ophthalmic & 19 \\
maxillary & 6 \\
Otic & mandibular & 2 \\
Cervical & & 3 \\
Thoracic & & 14 \\
Lumbosacral & & 45 \\
& & 9 \\
\hline
\end{tabular}

over hours in 4 patients and over days in 7 . The degree of disability was graded as mild in 1 , moderate in 6 anf severe in 4.

Computed tomographic (CT) scan of the brain was performed in 3 patients, being normal in patient 1 ; with delayed contralateral hemiparesis, and patient 2 , with third nerve palsy, but showing mild cerebral oedema in patient 11 , who had meningitis. Cerebral angiography in the patient with delayed contralateral hemiparesis showed carotid siphon stenosis. Myelography was performed in patient 9 with acute spinal cord syndrome and this was normal. The cerebrospinal fluid (CSF) showed changes consistent with aseptic meningitis in patient 11 , but was normal in the 2 patients with myelitis (patients 9 and 10) and in patient 2. Electromyographic (EMG) changes of denervation were present in all 3 patients $(6,7$ and 8$)$ with segmental limb paresis.

A 4 week course of steroid therapy was given to patient 1 with delayed contralateral hemiparesis. The other 10 patients were given supportive treatment only. They did not receive any steroid or antiviral therapy. Eight patients made a complete recovery in a period ranging from 1 week to 9 months, with the majority having recovered within 3 months. Two patients had partial recovery and one was lost to follow-up.

Intravenous vidarabine $(10 \mathrm{mg} / \mathrm{kg} /$ day for one week) was given to 7 patients and intravenous acyclovir $\left(500 \mathrm{mg} / \mathrm{m}^{2}\right.$ every 8 hours for one week) to 2 patients. These 9 patients were immunocompromised 
with either a haematological malignancy or SLE. Cutaneous spread, visceral dissemination or neurological complications did not occur in any of them.

\section{Discussion}

Herpes zoster is a common cutaneous infection and is due to the reactivation of the varicella-zoster virus in the posterior root ganglion. ${ }^{1}$ The exact incidence in Hong Kong is not known, but in a large English series, the incidence was reported as 3.4 per $1,000,{ }^{1}$ being higher in immunocompromised patients. It usually affects the elderly but no age group is spared. The age distribution and the pattern of involvement in our patient population are similar to other reported series. ${ }^{1,2}$ However, our patient population belongs to a highly selected group as we have included only hospitalized subjects. It is therefore obvious that we have more patients with underlying malignancy or other predisposing systemic diseases. Furthermore, $8.6 \%$ of our patients developed zoster eruption whilst inpatients receiving specific therapy for their underlying problems. It is also interesting to note a female preponderance in our series, which probably reflects the predominance of females in our elderly population.

Motor complications are uncommon and occur in $5 \%$ of all cases of herpes zoster. ${ }^{3}$ Our higher figure of $11.8 \%$ probably reflects selection bias as patients having complications are more likely to be hospitalized. Of cranial nerve involvement, the most widely known is the Ramsay-Hunt syndrome with facial nerve paralysis. Other cranial nerves are seldom involved. ${ }^{2}$ Third nerve palsy associated with ophthalmic zoster is a rare but well-recognized condition. ${ }^{4,5,6}$ We encountered one case in our series. Although the associated diabetes mellitus may have caused the third nerve palsy, the temporal relationship with the infection and the pupillary involvement would favour this being a complication of ophthalmic zoster.

Segmental limb paresis occurred in $3.2 \%$ of patients in this series which is comparable to the $2.5 \%$ figure in a large series by Thomas \& Howard with 1,210 patients. ${ }^{2}$ In their series, the incidence of underlying malignancy was 3 times higher in patients with segmental zoster paresis than in those with cutaneous zoster alone, while none of our 3 patients had any underlying malignancy. The onset of weakness is usually acute and occurs within days of the cutaneous rash. Occasionally it may precede the cutaneous rash and the diagnosis may then be difficult. ${ }^{7}$ Muscle weakness usually occurs in the same segments as the cutaneous rash but weakness in less than $10 \%$ of cases may also occur in widely separated segments. ${ }^{2}$ Paresis is more common with the upper than the lower limbs.
Although neurogenic changes are demonstrated by EMG, it is uncertain whether the pathology lies in the anterior horn cells or in the anterior nerve root because of the lack of detailed neuro-pathological examination. However, the frequent reversibility of the motor deficits would favour primary affliction of the nerve root rather than the nerve cells. ${ }^{8}$ With physiotherapy alone, ultimate recovery is good in $70-80 \%$ of cases although this may take a few months to a few years. All our 3 cases had excellent recovery within a period of 3 to 9 months, indicating the benign course of this motor complication.

Other neurological complications of zoster include encephalitis, meningitis, myelitis, polyneuritis and, rarely, delayed contralateral hemiparesis following ophthalmic zoster.

Herpes zoster myelitis is a rare complication. In the series of Thomas and Howard, there was only 1 patient out of $1,210 .^{2}$ The pathogenesis of the cord lesion is not fully understood as pathological confirmations are few and difficult to obtain. Direct viral invasion of the cord was demonstrated in one autopsy case. ${ }^{9}$ There was extensive serpentine necrosis of the cord extending through many segments. Moreover, typical nuclear inclusions were seen and the varicella-zoster virus was isolated from the destroyed cord. Apart from direct viral invasion of the cord, destruction by an autoimmune vasculitis has also been proposed as a pathogenetic mechanism. ${ }^{10,11}$ Spontaneous recovery is not un common. ${ }^{12}$ Our 2 patients recovered spontaneously in one week and 4 weeks respectively. However, relentless ascending myelitis ending in death has also been reported. ${ }^{9,11,13}$ A recent report described arrest of progression of myelitis in one case with intravenous vidarabine treatment ${ }^{14}$ and another reported improvement in one case, ${ }^{15}$ but the use of intravenous acyclovir in zoster myelitis has so far not been reported.

Fifty-one cases of herpes zoster ophthalmicus complicated by delayed contralateral hemiparesis were recently reviewed. ${ }^{16}$ Histological confirmation was obtained in 14 patients; the diagnosis in the remainder was based on circumstantial evidence. It is postulated that a direct spread of the virus along the ophthalmic division of the trigeminal nerve to the wall of ipsilateral main proximal branches of the circle of Willis results in necrotizing angiitis with thrombosis, ${ }^{17,18}$ although in situ thrombosis without inflammatory vasculitis has also been reported. ${ }^{19}$ The mortality is around $20 \%$, being especially high among those with clinical features of meningoencephalitis or diffuse granulomatous angiitis. ${ }^{20}$ We encountered only one patient with delayed contralateral hemiparesis among 19 with ophthalmic zoster. Although there was no histological confirmation, the temporal relationship to the ophthalmic zoster, the lack of risk factors for cerebral thrombosis and the 
angiographic demonstration of siphon stenosis would favour this being a complication of the zoster infection. This patient did not have signs of diffuse involvement and he made a good recovery.

For immunocompromised patients, intravenous vidarabine or acyclovir has been shown to be effective in preventing potentially fatal cutaneous and visceral dissemination. ${ }^{21,22}$ Therapy administered within 72 hours of the onset of cutaneous zoster confers the greatest benefit. Our 9 immunocompromised patients were treated ( 7 with vidarabine and 2 with acyclovir) and none developed cutaneous or visceral spread. Specific anti-viral treatment is therefore indicated in these patients and acyclovir is preferred to vidarabine because of its greater efficacy in zoster infection ${ }^{23}$ and better solubility. Moreover, the relative lack of renal or marrow toxicity renders acyclovir particularly suitable for patients with concomitant renal or

\section{References}

1. Hope-Simpson, R.E. The nature of herpes zoster: A long term study and a new hypothesis. Proc Roy Soc Med 1965, 58: 9-20.

2. Thomas, J.E. \& Howard, F.M. Segmental zoster paresis - a disease profile. Neurology (Minneap) 1972, 22: $459-466$.

3. Grant, B.D., Rowe, C.R. Motor paralysis of the extremities in herpes zoster. J Bone Joint Surg 1961, 43A: 885-896.

4. Godtfredsen, E. Pathogenesis of cranial nerve lesions, notably ophthalmoplegias, complicating herpes zoster ophthalmicus. Acta Psychiat (Kbh) 1948, 23: 69-77.

5. Kendall, D. Motor complications of herpes zoster. $\mathrm{Br}$ Med J 1957, 2: 616-618.

6. Payten, R.J. \& Dawes, J.D.K. Herpes zoster of the head and neck. $J$ Laryngol Otol 1972, 86: 1031-1055.

7. Taterka, J.H. \& O'Sullivan, M.E. The motor complication of herpes zoster. JAMA 1943, 122: 737-739.

8. Schliack, H. \& Schnedider, H. Segmentale motorische Paresen beim Zoster. Deutsch Med Wschr 1969, 94: 1861-1866.

9. Hogan, E.L. \& Krigman, M.R. Herpes zoster myelitis: Evidence for viral invasion of spinal cord. Arch Neurol 1973, 29: 309-313.

10. Applebaum, E., Kreps, S.I. \& Sunshine, A. Herpes zoster encephalitis. Am J Med 1962, 32: 25-31.

11. Rose, F.C., Brett, E.M. \& Burston, J. Zoster encephalomyelitis. Arch Neurol 1964, 11: 155-172.

12. Harrison, R.J. Zoster myelitis presenting with acute retention of urine. Proc Roy Soc Med 1964, 57: 589-590.

13. Cole, M. Myelopathy associated with herpes zoster. Rocky Mt Med J 1978, 75: 317-319.

14. Muder, R.R. \& Lumish, R.M. Myelopathy after herpes zoster. Arch Neurol 1983, 40: 445-446.

15. Corston, R.N., Logsdail, S. \& Godwin-Austen, R.B. Herpes zoster myelitis treated successfully with vidarabine. $\mathrm{Br}$ Med J 1981, 283: 698-699.

16. Reshef, E., Greenberg, S.B. \& Jankovic, J. Herpes zoster ophthalmicus followed by contralateral hemiparesis: haematological diseases, although judicious use of this drug with appropriate dosage adjustment must be emphasized.

In immunocompetent patients, intravenous acyclovir improves the rate of cutaneous healing and decreases pain in acute zoster although it does not decrease the incidence of post-herpetic neuralgia. ${ }^{24,25}$ With its high cost and inconvenience of administration, routine use of intravenous acyclovir in immunocompetent subjects with herpes zoster is hard to justify. Oral acyclovir, still under clinical trial, ${ }^{26,}{ }^{27}$ may, however, have a promising role in the future.

\section{Acknowledgement}

We wish to thank Mrs Shirla Tam and Mrs Monica Chan for secretarial assistance. report of two cases and review of literature. $J$ Neurol Neurosurg Psychiatry 1985, 48: 122-127.

17. Linnemann, C.C. \& Alvira, M.M. Pathogenesis of varicella-zoster angiitis in the CNS. Arch Neurol 1980, 37: 239-240.

18. Gasperetti, C. \& Song, S.K. Contralateral hemiparesis following herpes zoster ophthalmicus. J Neurol Neurosurg Psychiatry 1985, 48: 338-341.

19. Eidelberg, D., Sotrel, A., Horoupian, D.S., Neumann, P.E., Pumarola-Sune, T. \& Price, R.W. Thrombotic cerebral vasculopathy associated with herpes zoster. Ann Neurol 1986, 19: 7-14.

20. Rosenblum, W.L. \& Hadfield, M.G. Granulomatous angiitis of nervous system in cases of herpes zoster and lymphosarcoma. Neurology (Minneap) 1972, 22: 348-354.

21. Whitley, R.J., Soong, S.J. \& Dolin, R. Early vidarabine therapy to control the complications of herpes zoster in immunosuppressed patients. $N$ Eng $J$ Med 1982, 307: 971-975.

22. Balfour, H.H., McMonigal, K.A. \& Bean, B. Acyclovir therapy of varicella-zoster virus infections in immunocompromised patients. J Antimicrob Chemother 1983, 12: (Suppl. B): 169-179.

23. Shepp, D.H., Dandliker, P.S. \& Meyers, J.D. Treatment of varicella-zoster virus infection in severely immunocompromised patients. $N$ Engl $J$ Med 1986, 314: 208-212.

24. Peterslund, N.A., Seyer-Hansen, K., Ipsen, J., Esmann, V., Schonheyder, H., Juhl, H. Acyclovir in herpes zoster. Lancet 1981, ii: 827-830.

25. Bean, B., Braun, C., \& Balfour, H.H. Jr. Acyclovir therapy for acute herpes zoster. Lancet 1982, ii: 118-121.

26. Finn, R. \& Smith, M.A. Oral acyclovir for herpes zoster. Lancet 1984, ii: 575.

27. McKendrick, M.W., McGill, J.I., Bell, A.M., Hickmott, E. \& Burke, C. Oral acyclovir for herpes zoster. Lancet 1984, ii: 925. 